\title{
Physical properties of the Sh2-104 H II region as seen by Herschel ${ }^{\star}$
}

\author{
J. A. Rodón ${ }^{1}$, A. Zavagno ${ }^{1}$, J.-P. Baluteau ${ }^{1}$, L. D. Anderson ${ }^{1}$, E. Polehampton ${ }^{2,3}$, A. Abergel ${ }^{4}$, F. Motte ${ }^{5}$, \\ S. Bontemps ${ }^{5,6}$, P. Ade ${ }^{16}$, P. André ${ }^{\text {, H. Arab }}{ }^{4}$, C. Beichman ${ }^{8}$, J.-P. Bernard ${ }^{7}$, K. Blagrave ${ }^{14}$, F. Boulanger ${ }^{4}$, M. Cohen ${ }^{9}$, \\ M. Compiegne ${ }^{14}$, P. Cox ${ }^{10}$, E. Dartois ${ }^{4}$, G. Davis ${ }^{11}$, R. Emery ${ }^{16}$, T. Fulton ${ }^{19}$, C. Gry ${ }^{1}$, E. Habart ${ }^{4}$, M. Halpern ${ }^{13}$, \\ M. Huang ${ }^{11}$, C. Joblin ${ }^{7}$, S. C. Jones ${ }^{2}$, J. Kirk ${ }^{16}$, G. Lagache ${ }^{4}$, T. Lin ${ }^{3}$, S. Madden ${ }^{5}$, G. Makiwa ${ }^{2}$, P. Martin ${ }^{14}$, \\ M.-A. Miville-Deschênes ${ }^{4}$, S. Molinari ${ }^{15}$, H. Moseley ${ }^{18}$, D. Naylor ${ }^{2}$, K. Okumura ${ }^{5}$, F. Orieux ${ }^{12}$, \\ D. Pinheiro Gonçalves ${ }^{14}$, T. Rodet $^{12}$, D. Russeil ${ }^{1}$, P. Saraceno ${ }^{15}$, S. Sidher ${ }^{3}$, L. Spencer ${ }^{2}$, \\ B. Swinyard ${ }^{3}$, D. Ward-Thompson ${ }^{16}$, and G. White ${ }^{17,20}$
}

(Affiliations are available in the online edition)

Received 31 March 2010 / Accepted 7 May 2010

\begin{abstract}
Context. Sh2-104 is a Galactic H II region with a bubble morphology, detected at optical and radio wavelengths. It is considered the first observational confirmation of the collect-and-collapse model of triggered star-formation.

Aims. We aim to analyze the dust and gas properties of the Sh2-104 region to better constrain its effect on local future generations of stars. In addition, we investigate the relationship between the dust emissivity index $\beta$ and the dust temperature, $T_{\text {dust }}$.

Methods. Using Herschel PACS and SPIRE images at 100, 160, 250, 350 and $500 \mu \mathrm{m}$ we determine $T_{\text {dust }}$ and $\beta$ throughout Sh2-104, fitting the spectral energy distributions (SEDs) obtained from aperture photometry. With the SPIRE Fourier-transform spectrometer (FTS) we obtained spectra at different positions in the Sh2-104 region. We detect $J$-ladders of ${ }^{12} \mathrm{CO}$ and ${ }^{13} \mathrm{CO}$, with which we derive the gas temperature and column density. We also detect proxies of ionizing flux as the $[\mathrm{NII}]{ }^{3} P_{1}-{ }^{3} P_{0}$ and $[\mathrm{CI}]{ }^{3} P_{2}-{ }^{3} P_{1}$ transitions.

Results. We find an average value of $\beta \sim 1.5$ throughout Sh2-104, as well as a $T_{\text {dust }}$ difference between the photodissociation region (PDR, $\sim 25 \mathrm{~K}$ ) and the interior $(\sim 40 \mathrm{~K})$ of the bubble. We recover the anti-correlation between $\beta$ and dust temperature reported numerous times in the literature. The relative isotopologue abundances of CO appear to be enhanced above the standard ISM values, but the obtained value is very preliminary and is still affected by large uncertainties.
\end{abstract}

Key words. stars: formation - ISM: bubbles - H II regions - dust, extinction - infrared: ISM - ISM: individual objects: Sh2-104

\section{Introduction}

Sharpless 104 (Sh2-104, Sharpless 1959) is an optically visible Galactic H II region with a bubble morphology, excited by an O6V star (Crampton et al. 1978; Lahulla 1985). It is located $\sim 4 \mathrm{kpc}$ from the Sun (Deharveng et al. 2003), with galactic coordinates 74.7620; +0.60 (J2000).

Deharveng et al. (2003) proposed Sh2-104 as a strong candidate for massive triggered star formation through the collect-and-collapse process (Elmegreen \& Lada 1977). The ionized region is also visible at radio wavelengths (Fich 1993), and an ultracompact (UC) HII region, coincidant with the IRAS $20160+3636$ source, lies at its eastern border (Condon et al. 1998).

We present new submm images and spectra taken towards Sh2-104 with the Herschel Space Observatory (Pilbratt et al. 2010). These observations allow us to map a wavelength range not easily accessed before, providing new insights into the dust and gas properties of Sh2-104.

* Herschel is an ESA space observatory with science instruments provided by European-led Principal Investigator consortia and with important participation from NASA.

\section{Observations}

The Herschel observations were taken on 2009 December 17 simultaneously with PACS (Poglitsch et al. 2010) and SPIRE (Griffin et al. 2010), as part of the guaranteed-time key-projects "Evolution of Interstellar Dust" of SPIRE (Abergel et al. 2010), and HOBYS of PACS (Motte et al. 2010). A $15^{\prime} \times 15^{\prime}$ region was imaged with PACS at 100 and $160 \mu \mathrm{m}$ (at resolutions of $10^{\prime \prime}$ and $14^{\prime \prime}$ ), and with SPIRE at 250,350 and $500 \mu \mathrm{m}$ (at resolutions of $18^{\prime \prime}, 25^{\prime \prime}$ and $36^{\prime \prime}$ ). Spectra were taken with the SPIRE-FTS long and short wavelength receivers (SLW and SSW, respectively) at seven different positions with sparse sampling, covering the $194-671 \mu \mathrm{m}$ range. The resolution at the receivers' central pixels varies between 17-19" for SSW and 29-42" for SLW. The data were reduced with the HIPE software version 2.0 with the latest standard calibration (Swinyard et al. 2010).

Figure 1 shows a color-composite image of Sh2-104 with PACS $100 \mu \mathrm{m}$ (blue), SPIRE $250 \mu \mathrm{m}$ (green) and SPIRE $500 \mu \mathrm{m}$ (red). Different regions of interest, addressed in the following sections, are superimposed. We can see that the interior of the bubble is brighter in the PACS band, showing the hotter temperatures of the material in this region. On the other hand, outside 


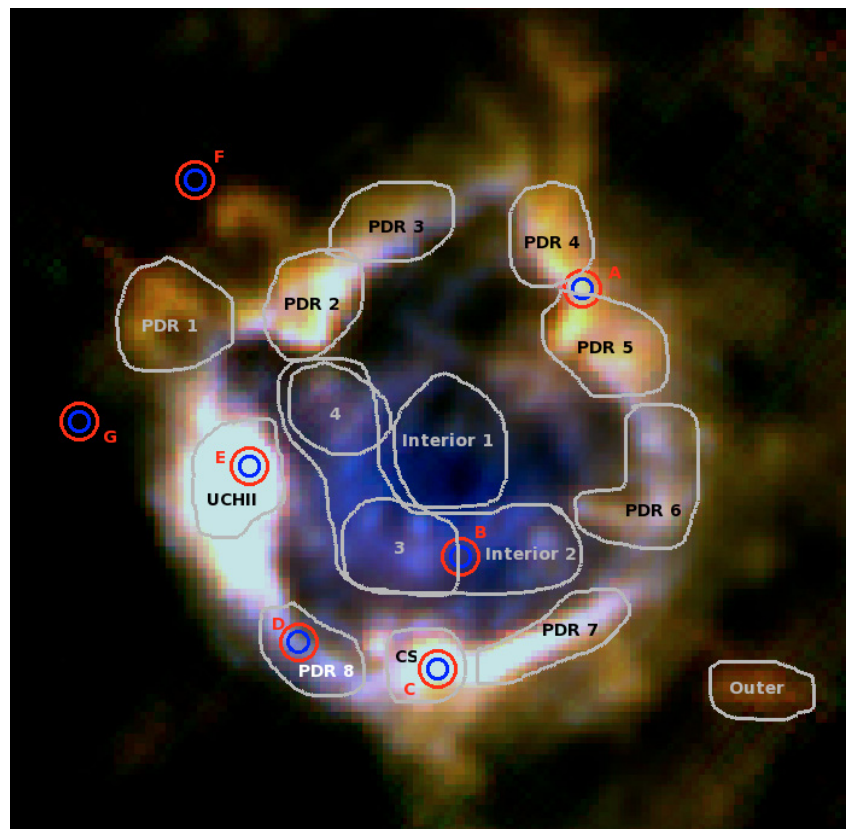

Fig. 1. Composite image of Sh2-104. The field is $\sim 13^{\prime} \times 13^{\prime}$ and shows PACS $100 \mu \mathrm{m}$ emission in blue, SPIRE $250 \mu \mathrm{m}$ in green and SPIRE $500 \mu \mathrm{m}$ in red. Outlined are the regions where aperture photometry was applied, the region used for background substraction is off the map. Blue and red circles mark the pointings of the central pixels of SPIREFTS SSW and SLW, respectively. Their diameter represents their respective average resolution of $19^{\prime \prime}$ and $35^{\prime \prime}$.

the bubble the material is colder and emits stronger in the SPIRE bands.

\section{Dust properties}

We assume the dust emission in Sh2-104 can be modeled by an (optically thin) gray-body and that the emissivity of the dust grains can be fitted with a power law

$S_{v} \propto \Omega B_{v}(T) \kappa_{0}\left(\frac{v}{v_{0}}\right)^{\beta} N$,

where $S_{v}$ is the measured flux density at frequency $v, \Omega$ is the observing beam solid angle, $B_{v}(T)$ is the Planck function for temperature $T$ at frequency $v, \kappa_{0}\left(v / v_{0}\right)^{\beta}$ is the dust opacity, $\beta$ is the dust emissivity index, and $N$ is the dust column density (Hildebrand 1983). The value of $\beta$ is believed to range between $\sim 1$ and $\sim 2$, but is an open issue that is still discussed, as is its dependence on the dust temperature (see e.g., Ossenkopf \& Henning 1994). Dupac et al. (2003) and Désert et al. (2008) found an inverse relationship between $\beta$ and $T_{\text {dust }}$. On the other hand, Shetty et al. (2009) suggested that this result arises from noise and the combination of multiple emission components along the line of sight.

To determine the dust temperature structure of Sh2-104, we performed aperture photometry measurements on selected areas in the field. The apertures are shown in Fig. 1 and sample the interior of the bubble and the photodissociation region (PDR), including the UCH II region associated with IRAS 20160+3636. We used a single aperture (not shown) to account for the background emission. We fitted the PACS+SPIRE emission for all regions. These data represent the cold emission component, therefore we fitted a single temperature. We did this first allowing $\beta$ to vary, and later fixing $\beta=1.5$. The resulting $T_{\text {dust }}$ and
Table 1. Results from aperture photometry.

\begin{tabular}{|c|c|c|c|}
\hline Region & $\begin{array}{r}T_{\text {dust }}(\mathrm{K}) \\
\beta\end{array}$ & $\beta$ & $\begin{array}{c}T_{\text {dust }}(\mathrm{K}) \\
\beta=1.5\end{array}$ \\
\hline $\mathrm{CS}$ & $25 \pm 3$ & $1.5 \pm 0.3$ & $25.5 \pm 0.9$ \\
\hline Interior 1 & $43 \pm 12$ & $1.2 \pm 0.4$ & $35.7 \pm 2.1$ \\
\hline Interior 2 & $39 \pm 9$ & $1.3 \pm 0.3$ & $33.5 \pm 1.7$ \\
\hline Interior 3 & $47 \pm 15$ & $1.0 \pm 0.4$ & $34.2 \pm 1.9$ \\
\hline Interior 4 & $35 \pm 8$ & $1.3 \pm 0.4$ & $30.4 \pm 1.4$ \\
\hline Outer & $17 \pm 2$ & $1.7 \pm 0.4$ & $17.3 \pm 0.4$ \\
\hline PDR 1 & $20 \pm 2$ & $1.7 \pm 0.4$ & $21.7 \pm 0.6$ \\
\hline PDR 2 & $22 \pm 3$ & $1.7 \pm 0.3$ & $24.1 \pm 0.8$ \\
\hline PDR 3 & $23 \pm 3$ & $1.7 \pm 0.4$ & $24.6 \pm 0.8$ \\
\hline PDR 4 & $22 \pm 3$ & $1.5 \pm 0.3$ & $22.3 \pm 0.7$ \\
\hline PDR 5 & $22 \pm 3$ & $1.5 \pm 0.3$ & $22.9 \pm 0.7$ \\
\hline PDR 6 & $24 \pm 3$ & $1.8 \pm 0.3$ & $26.0 \pm 0.9$ \\
\hline PDR 7 & $25 \pm 3$ & $1.7 \pm 0.3$ & $26.9 \pm 1.0$ \\
\hline PDR 8 & $26 \pm 4$ & $1.7 \pm 0.4$ & $28.6 \pm 1.2$ \\
\hline UCHII & $29 \pm 5$ & $1.8 \pm 0.3$ & $32.7 \pm 1.5$ \\
\hline
\end{tabular}

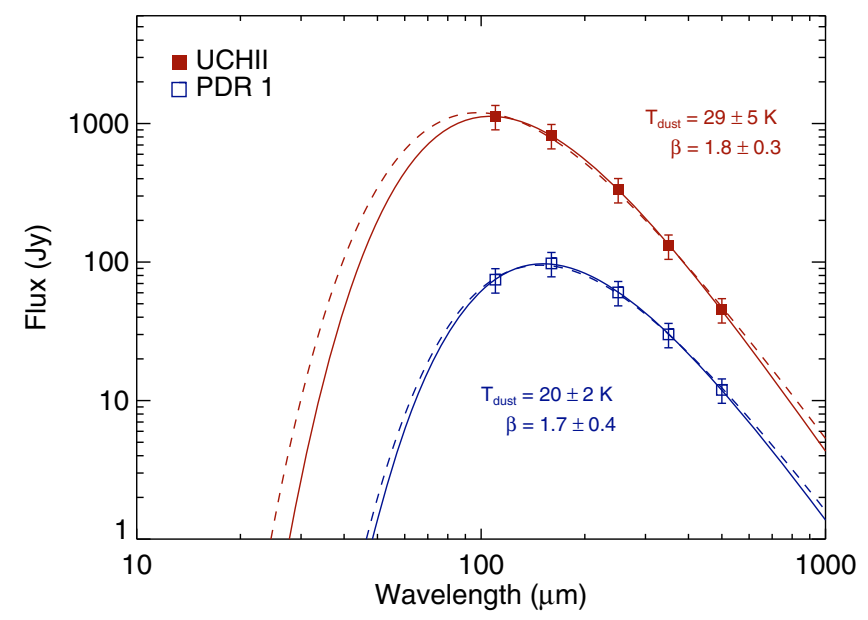

Fig. 2. Example of the SEDs obtained for regions UCHII (filled red squares) and PDR 1 (open blue squares). A dashed line is the fit with $\beta=1.5$ and a solid line is the fit with $\beta$ allowed to vary, with the resulting $\left(T_{\text {dust }} ; \beta\right)$ values shown in the respective color. The temperature difference between the regions is apparent, as is the difference in the peak sampling of the SED for PDR 1 and UCHII.

$\beta$ values are shown in Table 1 , their uncertainties are the formal $1 \sigma$ values from the fitting procedure. Figure 2 shows examples of the fitted spectral energy distributions (SEDs) for the regions $U C H I I$ and PDR 1. Apart from the clear difference in $T_{\text {dust }}$, it can be seen that for UCHII the PACS+SPIRE emission does not allow to sample the peak of the SED, which is reflected in a larger error in the fit. This is also seen in the four Interior \# regions, suggesting the presence of a warmer emission component that contributes to the shorter- $\lambda$ emission. Observations in the mid-IR can provide a constraint on this component, and a 2-temperatures fitting would be more appropriate to determine the $T_{\text {dust }}$ and $\beta$ values.

The temperatures throughout the PDR are between $\sim 20$ and $\sim 30 \mathrm{~K}$, and the UCH II region is marginally warmer. The region outside the bubble (region Outer) is the coldest, while the regions mapping the interior of the bubble are hotter, with an average temperature of $\sim 40 \mathrm{~K}$. The average $\beta$ obtained is $\sim 1.5$, therefore the temperatures obtained from the fit with a fixed $\beta=1.5$ do not significantly differ from those obtained with a free $\beta$ (see Table 1$)$. 
J. A. Rodón et al.: Physical properties of the Sh2-104 H II region as seen by Herschel

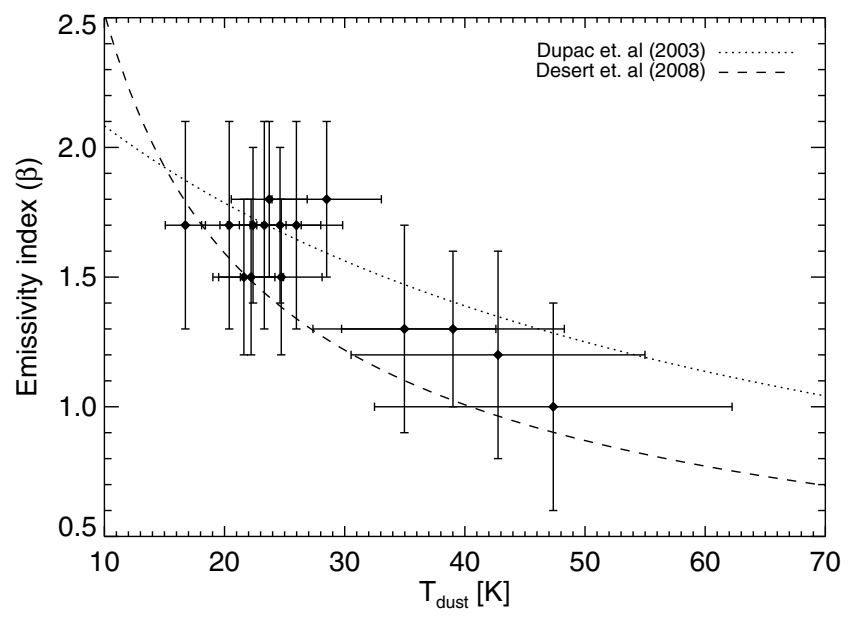

Fig. 3. Distribution of the emissivity index $\beta$ vs. dust temperature. The dotted and dashed lines are the relationships found by Dupac et al. (2003) and Désert et al. (2008), respectively. We cannot distinguish between the two relationships, but lower $\beta$ values correspond to higher temperatures (see text).

Figure 3 shows the distribution of the spectral indices $\beta$ vs. dust temperature, along with the relationships found by Dupac et al. (2003) and Désert et al. (2008). Within the uncertainties, our results agree with both relationships. We can also identify two groups, one with temperatures between 35 and $47 \mathrm{~K}$ and an emissivity index between 1.0 and 1.3, and the other with $T=17-29 \mathrm{~K}$ and $\beta=1.5-1.8$. These two groups show that on average higher $\beta$ values are preferentially associated with colder material.

Although anti-correlation between $\beta$ and $T_{\text {dust }}$ is reported in the literature (e.g., Dupac et al. 2003; Yang \& Phillips 2007; Désert et al. 2008), it is yet not clear which physical processes are behind it. Other authors studying this relationship examine the emission from different regions scattered in the sky. In contrast, we are finding this anti-correlation in the analysis of one contiguous complex object at a specific location in the sky. A $\beta-T_{\text {dust }}$ relationship may indicate a change of the dust properties (Stepnik et al. 2003). Grain fluffiness in particular increases the emissivity index while keeping a relatively low temperature (e.g., Stognienko et al. 1995; Fogel \& Leung 1998).

Fluffy grains result from grain coagulation and growth. The grain coagulation timescale and feasibility depend on factors such as the existence of ice mantles, grain size, and relative grain velocities.

We are finding the highest $\beta$ values and lowest $T_{\text {dust }}$ values toward the PDR of Sh2-104, which would imply then that the fluffiest and largest grains are located in the PDR. The question remains whether the PDR dust coagulated after the creation of the $\mathrm{H}$ II region, or if the birth of the $\mathrm{H}$ II region has destroyed the already coagulated dust located in the ionized cavity.

\section{Gas properties}

The seven pointings of the central pixels observed with SPIREFTS are marked with red (SLW) and blue (SSW) circles in Fig. 1. In total we detected the transitions described in Table 2. The richest spectrum (Fig. 4) was obtained towards pointing $E$, which targets the UCH II region associated with IRAS $20160+3636$. The most prominent features are the $\mathrm{CO} J$-ladder and the [NII] ${ }^{3} P_{1}-{ }^{3} P_{0}$ transition, an important ionized-gas coolant and a proxy for the $\mathrm{H} \alpha$ flux (see e.g., Oberst et al. 2006).
Table 2. Lines detected with SPIRE-FTS in the different pointings.

\begin{tabular}{lcc|lcc}
\hline \hline Transition & Rest $\lambda(\mu \mathrm{m})$ & $E_{\text {up }}(\mathrm{K})$ & Transition & Rest $\lambda(\mu \mathrm{m})$ & $E_{\text {up }}(\mathrm{K})$ \\
\hline $\mathrm{CO}(4-3)$ & 650.3 & 55.4 & ${ }^{13} \mathrm{CO}(5-4)$ & 544.2 & 79.4 \\
$\mathrm{CO}(5-4)$ & 520.2 & 83.0 & ${ }^{13} \mathrm{CO}(6-5)$ & 453.5 & 111.1 \\
$\mathrm{CO}(6-5)$ & 433.6 & 116.3 & ${ }^{13} \mathrm{CO}(7-6)$ & 388.7 & 148.2 \\
$\mathrm{CO}(7-6)$ & 371.7 & 155.0 & ${ }^{13} \mathrm{CO}(8-7)$ & 340.2 & 190.5 \\
$\mathrm{CO}(8-7)$ & 325.2 & 199.3 & ${ }^{13} \mathrm{CO}(9-8)$ & 302.4 & 238.1 \\
$\mathrm{CO}(9-8)$ & 289.1 & 249.1 & {$[\mathrm{CI}]{ }^{3} P_{1}-{ }^{3} P_{0}$} & 609.1 & 23.6 \\
$\mathrm{CO}(10-9)$ & 260.2 & 304.4 & {$[\mathrm{CI}]{ }^{3} P_{2}-{ }^{3} P_{1}$} & 370.4 & 47.3 \\
$\mathrm{CO}(11-10)$ & 236.6 & 365.3 & $\mathrm{CH}^{+}(1-0)$ & 359.0 & 40.1 \\
$\mathrm{CO}(12-11)$ & 216.9 & 431.7 & {$[\mathrm{NII}]{ }^{3} P_{1}-{ }^{3} P_{0}$} & 205.2 & 70.2 \\
$\mathrm{CO}(13-12)$ & 200.3 & 503.6 & & & \\
\hline
\end{tabular}

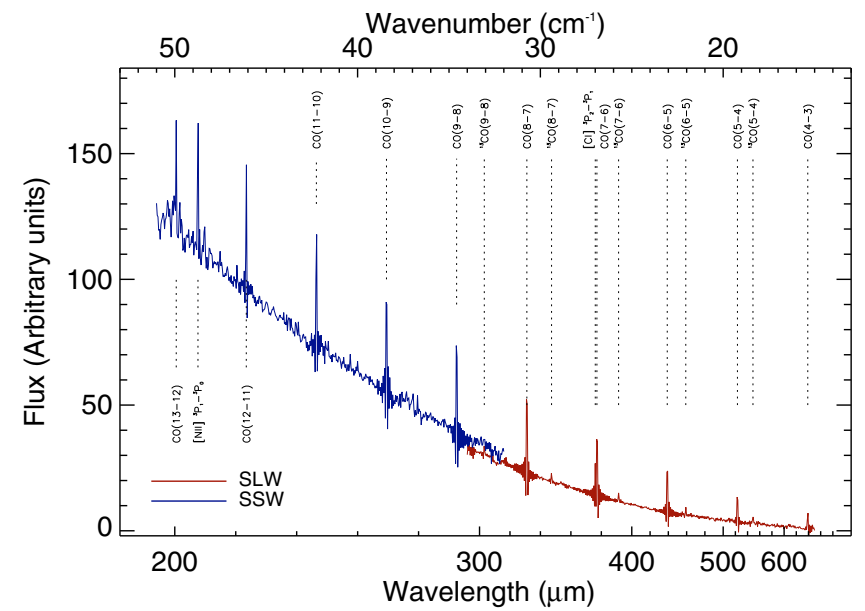

Fig. 4. SPIRE-FTS spectrum corresponding to the central pixels of SLW (pixel C3, red) and SSW (pixel D4, blue), towards the UCH II region associated with IRAS $20160+3636$ (pointing $E$ ). The lines detected are labeled. The jump between continuum levels is a calibration effect.

In the simple hypothesis of optically thin emission, we plotted ${ }^{12} \mathrm{CO}$ and ${ }^{13} \mathrm{CO}$ excitation diagrams, following the formulation of Johansson et al. (1984). An example is shown in Fig. 5 for pointing $E$. The slope of the linear fit for ${ }^{12} \mathrm{CO}$ results in a temperature of $T^{12}=246 \pm 2 \mathrm{~K}$, and $T^{13}=170 \pm 53 \mathrm{~K}$ for ${ }^{13} \mathrm{CO}$. The total column densities derived are $N_{T}^{12}=10^{16.36 \pm 0.01}$ and $N_{T}^{13}=10^{15.4 \pm 0.1} \mathrm{~cm}^{-2}$, respectively. The fits in Fig. 5 do not include all the measurements. The downturn seen in the lower levels of ${ }^{12} \mathrm{CO}$ is interpreted as the optically thick/thin regime turnover, and is most likely a real physical feature and not an instrumental or calibration effect, because it is only seen for that species and not, for example, for ${ }^{13} \mathrm{CO}$. If the lines were emitted from an optically thick medium, their intensity would be underestimated, thus their respective column densities would also be a lower limit. Therefore, the optically thin assumption would hold for ${ }^{12} \mathrm{CO}$ only for transitions higher than $J=9 \rightarrow 8$, and those are the points included in the fit.

For ${ }^{13} \mathrm{CO}$ on the other hand only the lines with wavelengths in the SPIRE-FTS SLW range are detected. These correspond to the $J=5 \rightarrow 4$ to $J=9 \rightarrow 8$ transitions. The ${ }^{13} \mathrm{CO}$ lines in the SPIRE-FTS SSW wavelenght range $(J=10 \rightarrow 9$ to $J=14 \rightarrow$ 13) are detected as upper limits, because the line positions are found displaced from their expected positions, indicating that we are probably seeing some "outlier" noise features rather than the lines themselves. Therefore, we fit and show in Fig. 5 only the five ${ }^{13} \mathrm{CO}$ transitions detected with SPIRE-FTS SLW. Following the reasoning of the previous paragraph, the ${ }^{13} \mathrm{CO}$ transitions are most likely optically thin. 


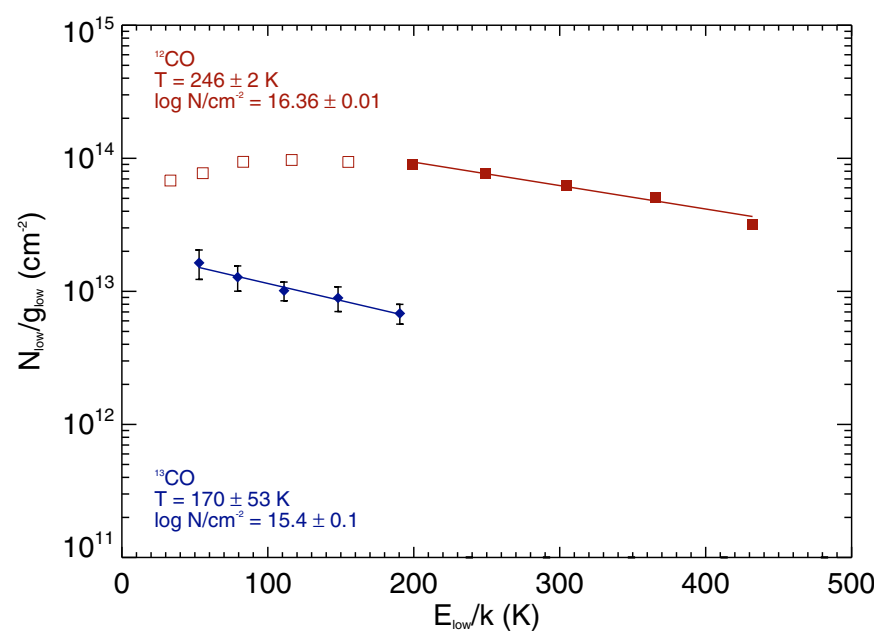

Fig. 5. ${ }^{12} \mathrm{CO}$ (red squares) and ${ }^{13} \mathrm{CO}$ (blue diamonds) rotational diagram for pointing $E$ towards the UCH II region. The solid lines are the best linear fit. Points included in the fit are shown as filled, points excluded as open. The turnover into the optically thick regime is noted in the lower-level transitions of ${ }^{12} \mathrm{CO}$.

The two distinct gas temperatures obtained with ${ }^{12} \mathrm{CO}$ and ${ }^{13} \mathrm{CO}$ suggest two different gas components or a stratification of the emitting region. The colder component is traced by the optically-thin ${ }^{13} \mathrm{CO}$ transitions at energy levels for which ${ }^{12} \mathrm{CO}$ is optically thick, while the hotter component is traced by the more energetic, optically-thin ${ }^{12} \mathrm{CO}$ transitions. Therefore it is likely that the colder gas is located at grater depths in the PDR than the hotter gas.

Assuming similar emitting volumes and beam filling factors as well as optically thin emission, our $\mathrm{CO}$ and ${ }^{13} \mathrm{CO}$ column density values imply an abundance ratio $\left[{ }^{12} \mathrm{CO}\right] /\left[{ }^{13} \mathrm{CO}\right]$, which is several times lower than the reported elemental value of ${ }^{12} \mathrm{C} /{ }^{13} \mathrm{C} \sim 69$ (Wilson 1999). This would imply an enhancement of the ${ }^{13} \mathrm{CO}$ isotopologue abundance.

However, several factors can contribute to the low ${ }^{12} \mathrm{C} /{ }^{13} \mathrm{C}$ abundance ratio we find. Perhaps the most important one would be the assumption of optically thin emission for ${ }^{12} \mathrm{CO}$. We used the high-energy transitions to derive its column density, and although in a first analysis they appear to be optically thin, it might not be the case (see e.g., Habart et al. 2010). To address this issue we will present and analyse PDR models of the Sh2-104 region in a forthcoming paper.

\section{Summary}

With Herschel PACS and SPIRE data we have analysed dust and gas properties of the bubble-shaped H II region Sh2-104. Aperture photometry of PACS+SPIRE images allowed us to derive the dust emissivity index $\beta$ and the dust temperature throughout Sh2-104. We found two different groups, one at colder temperatures and higher $\beta$, and the other at warmer temperatures and lower $\beta$. We recover the inverse $\beta-T_{\text {dust }}$ relationship reported in the literature, but the uncertainty in our fit prevents us from finding a precise depence. As an example, the different relationships found by Dupac et al. (2003) and Désert et al. (2008) are both compatible with our results. PACS and SPIRE have allowed us to constrain the "cold" side of the SED. We have estimated both $\beta$ and $T_{\text {dust }}$ simultaneously, but the uncertainties remain relatively high. With the data at hand we can only fit a single temperature component, which disregards the contribution of warmer components to the PACS shorter wavelengths. The existance of a $\beta-T_{\text {dust }}$ anti-correlation could be due to differences of the dust grain properties between the PDR and the H II cavity.

SPIRE-FTS spectra at different pointings throughout Sh2104 have unveiled the $\mathrm{CO}$ chemistry of the region in more detail. We detect the ${ }^{12} \mathrm{CO}$ and ${ }^{13} \mathrm{CO} J$-ladders up to the $J=13 \rightarrow 12$ and $J=9 \rightarrow 8$ transitions respectively, revealing the warm gas component in the region. Rotational diagrams towards the $\mathrm{UCH}$ II region in the PDR of Sh2-104 show that the ${ }^{13} \mathrm{CO}$ emission is optically thin and also the ${ }^{12} \mathrm{CO}$ for transitions above the $J=8$ level. The emission shows two different gas components, a colder one with a temperature of $\sim 170 \mathrm{~K}$ and a hotter one at a temperature of $\sim 250 \mathrm{~K}$. The $\mathrm{CO}$ column densities derived would suggest an enhancement of the ${ }^{13} \mathrm{CO}$ isotopologue abundance ratio with respect to the elemental value, but the uncertainties of the different assumptions are still too large to confirm that result. In a follow-up paper we will show models of the PDR of Sh2-104, which will provide better constraints on the gas temperature, density and column density structure.

Acknowledgements. SPIRE has been developed by a consortium of institutes led by Cardiff Univ. (UK) and including Univ. Lethbridge (Canada); NAOC (China); CEA, LAM (France); IFSI, Univ. Padua (Italy); IAC (Spain); Stockholm Observatory (Sweden); Imperial College London, RAL, UCL-MSSL, UKATC, Univ. Sussex (UK); Caltech, JPL, NHSC, Univ. Colorado (USA). This development has been supported by national funding agencies: CSA (Canada); NAOC (China); CEA, CNES, CNRS (France); ASI (Italy); MCINN (Spain); Stockholm Observatory (Sweden); STFC (UK); and NASA (USA). PACS has been developed by a consortium of institutes led by MPE (Germany) and including UVIE (Austria); KUL, CSL, IMEC (Belgium); CEA, LAM (France); MPIA (Germany); IFSI, OAP/AOT, OAA/CAISMI, LENS, SISSA (Italy); IAC (Spain). This development has been supported by the funding agencies BMVIT (Austria), ESA-PRODEX (Belgium), CEA/CNES (France), DLR (Germany), ASI (Italy), and CICT/MCT (Spain). Part of this work was supported by the ANR (Agence Nationale pour la Recherche) project "PROBeS", number ANR08-BLAN-0241.

\section{References}

Abergel, A., et al. 2010, A\&A, 518, L96

Benjamin, R. A., Churchwell, E., Babler, B. L., et al. 2003, PASP, 115, 953 Churchwell, E., Povich, M. S., Allen, D., et al. 2006, ApJ, 649, 759 Churchwell, E., Watson, D. F., Povich, M. S., et al. 2007, ApJ, 670, 428 Condon, J. J., Cotton, W. D., Greisen, E. W., et al. 1998, AJ, 115, 1693 Crampton, D., Georgelin, Y. M., \& Georgelin, Y. P. 1978, A\&A, 66, 1 Deharveng, L., Lefloch, B., Zavagno, A., et al. 2003, A\&A, 408, L25 Désert, F., Macías-Pérez, J. F., Mayet, F., et al. 2008, A\&A, 481, 411 Dupac, X., Bernard, J., Boudet, N., et al. 2003, A\&A, 404, L11

Elmegreen, B. G., \& Lada, C. J. 1977, ApJ, 214, 725

Fich, M. 1993, ApJS, 86, 475

Fogel, M. E., \& Leung, C. M. 1998, ApJ, 501, 175

Habart, E., et al. 2010, A\&A, 518, L116

Hildebrand, R. H. 1983, QJRAS, 24, 267

Griffin, M. J., et al. 2010, A\&A, 518, L3

Johansson, L. E. B., Andersson, C., Ellder, J., et al. 1984, A\&A, 130, 227

Lahulla, J. F. 1985, A\&AS, 61, 537

Motte, F., et al. 2010, A\&A, 518, L77

Oberst, T. E., Parshley, S. C., Stacey, G. J., et al. 2006, ApJ, 652, L125

Ossenkopf, V., \& Henning, T. 1994, A\&A, 291, 943

Pilbratt, G. L., et al. 2010, A\&A, 518, L1

Poglitsch, A., et al. 2010, A\&A, 518, L2

Sharpless, S. 1959, ApJS, 4, 257

Shetty, R., Kauffmann, J., Schnee, S., \& Goodman, A. A. 2009, ApJ, 696, 676

Stepnik, B., Abergel, A., Bernard, J., et al. 2003, A\&A, 398, 551

Stognienko, R., Henning, T., \& Ossenkopf, V. 1995, A\&A, 296, 797

Swinyard, B. M., et al. 2010, A\&A, 518, L4

Visser, R., van Dishoeck, E. F., \& Black, J. H. 2009, A\&A, 503, 323

Wilson, T. L. 1999, Reports on Progress in Physics, 62, 143

Yang, M., \& Phillips, T. 2007, ApJ, 662, 284 
1 Laboratoire d'Astrophysique de Marseille (UMR 6110 CNRS and Université de Provence), 38 rue F. Joliot-Curie, 13388 Marseille Cedex 13, France e-mail: jarodon@oamp.fr

2 Institute for Space Imaging Science, University of Lethbridge, Lethbridge, Canada

3 Space Science Department, Rutherford Appleton Laboratory, Chilton, UK

4 Institut d'Astrophysique Spatiale, CNRS/Université Paris-Sud 11, 91405 Orsay, France

5 CEA, Laboratoire AIM, Irfu/SAp, Orme des Merisiers, 91191 Gif-sur-Yvette, France

${ }^{6} \mathrm{CNRS} / \mathrm{INSU}$, Laboratoire d'Astrophysique de Bordeaux, UMR 5804, BP 89, 33271 Floirac Cedex, France

7 Universié de Toulouse; UPS; CESR; and CNRS; UMR5187, 9 avenue du colonel Roche, 31028 Toulouse Cedex 4, France

8 Infrared Processing \& Analysis Center, California Institute of Technology, Mail Code 100-22, 770 South Wilson Av, Pasadena, CA 91125, USA
9 University of California, Radio Astronomy Laboratory, Berkeley, 601 Campbell Hall, US Berkeley CA 94720-3411, USA

10 Institut de Radioastronomie Millimétrique (IRAM), 300 rue de la Piscine, 38406 Saint-Martin-d'Hères, France

11 National Astronomical Observatories, PR China

12 Laboratoire des Signaux et Systèmes (CNRS \& Supélec \& Université Paris-Sud 11), Moulon, 91192 Gif-sur-Yvette, France

13 Department of Physics and Astronomy, University of British Columbia, Vancouver, Canada

14 Canadian Institute for Theoretical Astrophysics, Toronto, Ontario, M5S 3H8, Canada

15 Istituto di Fisica dello Spazio Interplanetario, INAF, via del Fosso del Cavaliere 100, 00133 Roma, Italy

16 Department of Physics and Astronomy, Cardiff University, UK

17 Centre for Astrophysics and Planetary Science, School of Physical Sciences, University of Kent, Kent, UK

18 NASA - Goddard SFC, USA

19 Blue Sky Spectrosocpy Inc, Lethbridge, Canada

20 Department of Physics \& Astronomy, The Open University, Milton Keynes MK7 6AA, UK 